\title{
REFLEXIVE PRIMES, LOCALIZATION AND PRIMARY DECOMPOSITION IN MAXIMAL ORDERS
}

\author{
J. H. COZZENS AND F. L. SANDOMIERSKI
}

\begin{abstract}
If $R$ is a maximal order and $P$ a reflexive prime ideal of $R$, then the Goldie localization of $R$ at $P$ is shown to be the classical (partial) quotient ring of $R$ with respect to the Ore set $C(P)=\{r \in R \mid r x \in P \Rightarrow x \in P\}$. This is accomplished by introducing new symbolic powers of the prime $P$ which agree with Goldie's symbolic powers. As a consequence, whenever $P$ is a reflexive prime ideal of $R$ and $P^{(n)}$ the $n$th (Goldie) symbolic power of $P$, then an ideal $B$ is reflexive if and only if $B=\cap_{i=1}^{n} P_{i}^{\left(n_{i}\right)}$ for uniquely determined reflexive primes $P_{i}$ and integers $n_{i}>0$. More generally, each bounded essential right (left) ideal is shown to have a reduced primary decomposition and an explicit determination of the components is given in terms of the bound of the ideal.
\end{abstract}

1. In [67], Goldie gave a method for constructing a local ring $R_{P}$ given an arbitrary two-sided Noetherian ring $R$ and a prime ideal $P$, which for commutative $R$ coincides with the usual commutative localization at $P$. Just as in the commutative case, Goldie's localization at $P$ utilizes the set $C(P)$ $=\{x \in R \mid x+P$ is regular in $R / P\}$. There are many instances where $C(P)$ is an Ore set: $R$ any commutative Noetherian ring, $R$ an Asano order (Michler [69], Hajarnavis and Lenagan [71], Kuzmanovich [72]), $R$ a hereditary Noetherian prime ring with $P$ nonidempotent (Chatters and Ginn [72]), $R$ the enveloping algebra of finite dimensional nilpotent Lie algebra (McConnell [68]) and $R=A G, A$ a Noetherian prime ring of characteristic $0, G$ a finite group and $P$ the augmentation ideal of $R$ (Michler [72]).

The object of the first section of this paper is to show that whenever $R$ is a Noetherian maximal order and $P$ a reflexive prime, $C(P)$ is an Ore set of regular elements of $R$. Such rings include Asano orders and most "classical" maximal orders. To accomplish this, we first define a new symbolic power, denoted $P^{(n)}$, for a reflexive prime ideal of $R$. Actually, all of the results of this paper are valid for nonmaximal $R$ and reflexive primes $P$ satisfying $P^{*} P \neq P$ and $P P^{*} \subseteq R$. Next, we establish the basic properties of these symbolic powers, ultimately showing that they are identical to the $H_{n}$ of Goldie [67] (see also Michler [72] for another equivalent formulation). Although we use the

Received by the editors February 19, 1975 and, in revised form, October 20, 1975.

AMS (MOS) subject classifications (1970). Primary 16A08, 16A66; Secondary 16A10, 16A46.

Key words and phrases. Order, partial quotient ring, Ore set, equivalent order, maximal order, Asano order, reflexive module, symbolic power, associated prime, primary decomposition. 
equivalence of our definition and Goldie's to complete the proof of the main theorem, it is easy to prove this result directly using only the $P^{(n)}$ 's. For, by Small's theorem, $R / P^{(n)}$ trivially satisfies the Ore condition $\forall n$, a fact necessary for showing the Artin-Rees type condition of Goldie [67, Theorem 5.2]. However, we feel that the major thrust of this approach is the particularly simple, transparent description of the $H_{n}$ afforded by our assumptions.

Next, we show that the $P^{(n)}$ uniquely determine the reflexive ideals of $R$. More precisely, $B$ is a reflexive ideal, of $R$ if and only if $B=\cap_{i=1}^{m} P_{i}^{\left(n_{i}\right)}$ for uniquely determined reflexive primes $P_{i}$ and integers $n_{i} \geqslant 1$. In fact, the $P_{i}^{\left(n_{i}\right)}$ provide a reduced primary decomposition for $B$. As an immediate consequence, we show that each bounded, reflexive, essential right ideal has a primary decomposition and explicitly determine the factors in terms of its bound. This result generalizes analogous results for classical maximal orders due to Riley [65].

Throughout, $R$ will denote a fixed two-sided Noetherian semiprime ring which is a maximal order in its quotient ring $Q$. By Robson [68, Lemma 5.2], we can assume that $R$ is actually prime. If $I$ is any right $R$-module and $I^{*}=\operatorname{hom}_{R}(I, R)$, there is a canonical map $\varphi: I \rightarrow I^{* *}$ defined by $(f) \varphi(x)$ $=f(x) \forall x \in I, f \in I^{*}$. Whenever $\varphi$ is an isomorphism, $I_{R}$ is called reflexive. If $I_{R}$ is an essential $R$-submodule of $Q_{R}$, in particular, an essential right ideal of $R, I^{*}$ can be identified with the left $R$-submodule of $Q,\{q \in Q \mid q I \subseteq R\}$ and hence, $I$ can be identified with the right ideal $I^{* *}=\left\{q \in Q \mid I^{*} q \subseteq R\right\}$ whenever $I$ is reflexive. The two-sided ideal of $R, T=I^{*} I$ is called the trace ideal of $I$. Since $R$ is a maximal order, if $I$ is any two-sided ideal of $R,\{q \in Q \mid q I \subseteq R\}=\{q \in Q \mid I q \subseteq R\}$ and $O_{l}(I)=\{q \in Q \mid q I \subseteq I\}=R$ $=O_{r}(I)=\{q \in Q \mid I q \subseteq I\}$. Hence, for two-sided ideals $I$ of $R, I_{R}$ is reflexive if and only if ${ }_{R} I$ is reflexive. Thus, $I I^{*}$ is a two-sided ideal of $R$ as well.

Whenever $P$ is a prime ideal of $R, \mathscr{F}(P)=\left\{I_{R} \subseteq R_{R} \mid a^{-1} I \cap C(P)\right.$ $\neq \varnothing \forall a \in R\}\left(a^{-1} I=\{x \in R \mid a x \in I\}\right)$, where $\mathscr{F}=\mathscr{F}(P)$, is an idempotent topologizing family of right ideals of $R$ (see Goldie [67, p. 99]). The $\mathscr{F}$ closure of $I, \rho(I)$, is defined to be $\{r \in R \mid r J \subseteq I$ for some $J \in \mathscr{F}\}$. Whenever $C(P)$ is an Ore set, it is easily seen that $\rho(I)=\{r \in R \mid r c \in I$ for some $c \in C(P)\}$. Goldie [67] has shown that $\rho\left(P^{n}\right)=H_{n}$, the $n$th symbolic power of $P$.

The first lemma provides a useful module-theoretic characterization of reflexivity for an arbitrary essential right ideal of $R$.

LEMMA 1.1. Let $R$ be an order in $Q$. If $I_{R}$ is an essential right ideal of $R$, then $I_{R}$ is reflexive if and only if $(R / I)_{R}$ embeds monomorphically in a product of copies of the right $R$-module $(Q / R)_{R}$.

Proof. If $I=I^{* *}$ and $a \in R-I$ then there is an element $q \in I^{*}$ such that $q a \notin R$, hence $q$ induces by left multiplication an $R$-homomorphism $R / I \rightarrow Q / R$ which distinguishes $a+I \in R / I$ from 0 , thus $R / I$ embeds monomorphically in a product of copies of $Q / R$.

Conversely, if there is an embedding of $R / I$ in a product of copies of 
$Q / R, R / I \stackrel{\alpha}{\longrightarrow} \Pi Q / R$, then $\alpha$ followed by each projection $\Pi Q / R \rightarrow Q / R$ is given by some left multiplication by $q \in I^{*}$. Therefore, there is a subset $B \subseteq I^{*}$ such that if $a \in R$ and $B a \subseteq R$, then $a \in I$. Clearly then, if $a \in I^{* *}$ then $B a \subseteq I^{*} a \subseteq R$ so $a \in I$ and the lemma follows.

Definition. Let $P$ be a reflexive prime ideal of $R$. $P^{(n)}=\left\{r \in R \mid r P^{*}\right.$ $\left.\subseteq P^{(n-1)}\right\}$ where $P^{(0)}=R$, for $n=1,2,3, \ldots$

It is clear that $P^{(1)}=P$ and each $P^{(n)}$ is an ideal of $R$.

Proposition 1.2. Let $P$ be a reflexive prime ideal of $R$. Then:

(1) If $n=0,1,2, \ldots$ and $r \in R, r \in P^{(n+1)}$ if and only if $P^{*} r \subseteq P^{(n)}$.

(2) $P^{(n)}$ is a reflexive ideal of $R$ for $n=1,2,3, \ldots$

(3) $P^{n} \subseteq P^{(n)} \subsetneq P^{(n-1)}$ for $n=1,2,3, \ldots$

(4) $\cap_{n=1}^{\infty} P^{(n)}=0$.

(5) $P^{(n)}$ is $P$-primary for all $n \geqslant 1$, that is, if $A$ and $B$ are ideals of $R$ with $A B \subseteq P^{(n)}$, then $A \nsubseteq P(B \nsubseteq P) \Rightarrow B \subseteq P^{(n)}\left(A \subseteq P^{(n)}\right)$.

(6) $C(P)=C\left(P^{(n)}\right) \forall n$, where $C\left(P^{(n)}\right)=\left\{r \in R \mid r+P^{(n)}\right.$ is regular in $\left.R / P^{(n)}\right\}$.

Proof. We proceed by induction on $n$. The case $n=0$ being trivial. If $r P^{*} \subseteq P$ then $P^{*} r P^{*} P \subseteq P$ and since $P^{*} P \nsubseteq P$ by the maximality of $R$ and the reflexivity of $P, P^{*} r \subseteq P$. By symmetry (1) holds for $n=1$. Now observe that if (1) holds for all $k \leqslant n$ with $n \geqslant 1$ then $r P^{*} \subseteq P^{(n)}$ if and only if $P^{*} r P^{*} \subseteq P^{(n-1)}$. Thus, $r \in P^{(n+1)}$ if and only if $P^{*} r P^{*} \subseteq P^{(n-1)}$ and by the inductive assumption, if and only if $P^{*} r \subseteq P^{(n)}$.

The proof of (2) will be shown via induction on $n$. The case $n=1$ is true by hypothesis. Suppose $P^{(n)}$ is reflexive. If $r \in R-P^{(n+1)}$ then $P^{*} r \nsubseteq P^{(n)}$; thus it follows that $R / P^{(n+1)}$ embeds monomorphically as a right $R$-module in a product of copies of the right $R$-module $R / P^{(n)}$ using left multiplication by elements of $P^{*}$. It now follows from Lemma 1.1 and symmetry that $P^{(n+1)}$ is reflexive.

Next we show that $P^{n} \subseteq P^{(n)}$ for all $n \geqslant 1$ by induction on $n$. The case $n=1$ is obvious. Now suppose $P^{n} \subseteq P^{(n)}$; then since $P^{*} P^{n+1} \subseteq P^{n} \subseteq P^{(n)}$, we have $P^{n+1} \subseteq P^{(n+1)}$.

The inclusions $P^{(n)} \subseteq P^{(n-1)}$ are trivial as $1 \in P^{*}$. If $P^{(n)}=P^{(n-1)}$ then $P^{*} P^{(n)} \subseteq P^{(n-1)}=P^{(n)}$ and by the maximality of $R, P^{*} \subseteq R$ an impossibility, so (3) follows.

For the proof of (4) suppose $A=\cap_{n=1}^{\infty} P^{(n)} \neq 0$; then $A$ contains a regular element $d$, so

$$
\left(P^{(1)}\right)^{*} \varsubsetneqq\left(P^{(2)}\right)^{*} \varsubsetneqq \cdots \varsubsetneqq R d^{-1} \approx{ }_{R} R
$$

by (3) contradicting the assumption that $R$ is Noetherian.

Suppose $A B \subseteq P^{(n)}$ with $A \nsubseteq P$. Then $A B P^{*} \subseteq P^{(n-1)}$ and since $B \subseteq P$, $B P^{*} \subseteq P^{(n-1)}$ by induction $\Rightarrow B \subseteq P^{(n)}$. The other case where $B \nsubseteq P$ is handled similarly.

Finally, it is clear that $C(P) \subseteq \underline{C}\left(P^{(2)}\right) \subseteq \cdots$. If $c \in C\left(P^{(n+1)}\right)$ and 
$c x \in P^{(n)}, c x P \subseteq P^{(n+1)} \Rightarrow x P \subseteq P^{(n+1)} \Rightarrow x P P^{*} \subseteq P^{(n)}$. Since $P^{(n)}$ is $P$ primary and $P P^{*} \nsubseteq P, x \in P^{(n)}$. The proof of the left regularity of $c$ is identical.

Proposition 1.3. $P^{(n)}=H_{n} \forall n \geqslant 1$.

Proof. Clearly, $P^{(n)} \subseteq H_{n} \forall n$. By Goldie [67, Proposition 3.3], there exist ideals $F \in \mathscr{F}, G \in \mathcal{G}$ satisfying $G H_{n+1} F \subseteq P H_{n}$ which we can assume is contained in $P^{(n+1)}$ by induction. By definition of $P^{(n+1)}, G H_{n+1} F P^{*} \subseteq P^{(n)}$ $=H_{n}$. Since $H_{n+1} F \subseteq P, H_{n+1} F P^{*}$ is a two-sided ideal of $R$. Moreover, $G \nsubseteq P$ by definition of $\mathcal{G}$. Consequently, $H_{n+1} F P^{*} \subseteq P^{(n)}$ since $P^{(n)}=H_{n}$ is $P$-primary (Goldie [67, bottom of p. 95]), implying $H_{n+1} F \subseteq P^{(n+1)}$ by definition of $P^{(n+1)}$. By symmetry, $H_{n+1} \subseteq P^{(n+1)}$ completing the proof of the proposition.

THEOREM 1.4. If $R$ is a two-sided Noetherian prime ring which is a maximal order in its quotient ring and $P$ is a reflexive prime ideal of $R$, then $R$ satisfies the Ore condition with respect to $C(P)$ and its quotient ring (with respect to $C(P)$ ) is a Noetherian local ring.

Proof. By Goldie [67, Theorem 5.2], all that has to be shown is given any essential right ideal $E$ of $R$, there exists an $n>0$ such that $E \cap H_{n} \subseteq \rho(E P)$ where $\rho(E P)=\{x \in R \mid x F \subseteq E P$ for some $F \in \mathscr{F}\}$.

To demonstrate this, we borrow an argument used by Chatters and Ginn [72]. Thus, consider the right ideal

$$
I=\sum_{i \geqslant 1}\left(E \cap P^{(i)}\right)\left\{P^{-(i)}=\operatorname{hom}_{R}\left(P^{(i)}, R\right)\right\},
$$

which is finitely generated, say, $I=\sum_{i=1}^{n}\left(E \cap P^{(i)}\right) P^{-(i)}$,

$$
\begin{aligned}
\left(E \cap P^{(n+1)}\right) P^{-(n+1)} & \subseteq I \Rightarrow\left(E \cap P^{(n+1)}\right) T_{n+1} \\
& \subseteq I P^{(n+1)} \subseteq E P
\end{aligned}
$$

where $T_{n+1}=\operatorname{trace} P^{(n+1)}$. Since $T_{n} \nsubseteq P \forall n, x^{-1} T_{n+1} \cap C(P) \neq \varnothing \Rightarrow T_{n+1}$ $\in \mathscr{F}$, completing the proof.

2. The structure of reflexive ideals. Henceforth, $\mathscr{P}$ will denote the set of all reflexive primes of $R$. These primes are necessarily minimal by Cozzens and Sandomierski [75]. The following lemma lists some results which will be needed in the proof of 2.2 .

LEMMA 2.1. (1) If $I_{R}$ is an essential right ideal of $R$ and $P \in \mathscr{P}$, then $\left(I R_{P}\right)^{+}=R_{P} I^{*}$, where $\left(I R_{P}\right)^{+}$denotes the $R_{P}$ dual of $I R_{P}$. Moreover, $\left(I R_{P}\right)$ $\cap R=\rho(I)$ is a reflexive right ideal of $R$.

(2) If $\left\{B_{n} \mid n=1,2, \ldots\right\}$ is a sequence of reflexive ideals of $R$ with $B_{n} \supsetneqq B_{n+1}$ for all $n$, then $\cap B_{n}=0$. In addition, if $B$ is an ideal of $R$, then $B$ is contained in only finitely many $P \in \mathcal{P}$.

(3) If $B$ is an ideal of $R$ and $B \subseteq P \in \mathscr{P}$, then there is an index $n$ such that 
$B \subseteq P^{(n)}$ but $B \nsubseteq P^{(n+1)}$. Also $B R_{P} \cap R \nsubseteq P^{\prime}$ for all $P^{\prime} \in \mathscr{P}$ with $P^{\prime} \neq P$.

Proof. (1) Clearly $R_{P} I^{*} \subseteq\left(I R_{P}\right)^{+}$. If $q \in\left(I R_{P}\right)^{+}$then $q I \subseteq R_{P}$. Since $q I$ is a finitely generated right $R$-submodule of $R_{P}$ there is a $c \in C(P)$ such that $c q I \subseteq R$, hence $c q \in I^{*}$ and, therefore, $q \in R_{P} I^{*}$.

That $\left(I R_{P}\right) \cap R=\rho(I)$ is evident by previous remarks. Since $R_{P}$ is a bounded Asano order, $R_{P}$ is hereditary (Michler [69]), so $\rho(I) R_{P}$ is a finitely generated right ideal of $R_{P}$ and, therefore, $\rho(I) R_{P}=\left(\rho(I) R_{P}\right)^{++}=\rho(I)^{* *} R_{P}$ $=I R_{P}$ where the second equality follows from the first part and the third is evident, hence $\rho(I)^{* *} \subseteq \rho(I)$ and (1) follows.

(2) If $\cap B_{n} \neq 0$, then since $R$ is prime, $\cap B_{n}$ contains a regular element $d$; this gives rise to

$$
B_{I}^{*} \varsubsetneqq B_{2}^{*} \varsubsetneqq \cdots \varsubsetneqq B_{n}^{*} \varsubsetneqq \cdots \subseteq R d^{-1} \approx{ }_{R} R,
$$

contradicting the fact that $R$ is Noetherian, and the first part is shown.

Suppose $B$ is contained in infinitely many distinct $P \in \mathscr{P}$, say $P_{1}, P_{2}, \ldots, P_{n}$, $\ldots$ Let $B_{n}=P_{1} \cap \cdots \cap P_{n}$, then $B_{n}$ is reflexive by 1.1 , and $B_{n} \supsetneqq B_{n+1}$, since if $B_{n}=B_{n+1}, P_{1} P_{2} \cdots P_{n} \subseteq B_{n}=B_{n+1} \subseteq P_{n+1}$, an impossibility. Thus, $B \subseteq \cap B_{n}=0$ by the first part which is also absurd and (2) is verified.

(3) There is an index $n$ such that $B \subseteq P^{(n)}$ but $B \nsubseteq P^{(n+1)}$ since $\cap P^{(n)}=0$ by 1.2(4). Set $B_{P}=B R_{P}$ and $\bar{B}=B_{P} \cap R$. Clearly, $\bar{B}_{P}=B_{P}$ and since $\bar{B}_{P}$ is an ideal of the bounded Asano order $R_{P}, \bar{B}_{P}=P_{P}^{n}$ for some integer $n>0$. Consequently, $\bar{B}=P_{P}^{n} \cap R=P^{(n)}$ (see Goldie [67]). That $P^{(n)} \llbracket P^{\prime} \forall P^{\prime}$ $\in \mathscr{P}$ with $P^{\prime} \neq P$, is now clear.

THEOREM 2.2. If $B$ is a reflexive ideal of $R$ then $B$ is uniquely expressible as $B=P_{1}^{\left(n_{1}\right)} \cap \cdots \cap P_{k}^{\left(n_{k}\right)}$ with $P_{1}, \ldots, P_{k}$ distinct in $\mathcal{P}$. Furthermore, if $B \subseteq P$ $\in \mathcal{P}$, then $B R_{P} \cap R=P^{(n)}$ where $B \subseteq P^{(n)}$ but $B \nsubseteq P^{(n+1)}$.

Proof. By 2.1(2), $B$ is contained in only finitely many $P \in \mathcal{P}$, say $P_{1}, \ldots$, $P_{k}$. Let $n_{i}, i=1,2, \ldots, k$, be such that $B \subseteq P_{i}^{\left(n_{i}\right)}$ and $B \nsubseteq P_{i}^{\left(n_{i}+1\right)}$. Now $A=B\left(P_{1}^{-\left(n_{1}\right)}+\cdots+P_{k}^{-\left(n_{k}\right)}\right)$ is an ideal of $R$ and since $B P_{i}^{-\left(n_{i}\right)} \subseteq A, A$ is not contained in any $P \in \mathscr{P}$ by $2.1(3)$, hence $A^{*}=R$. Since

$$
\left(P_{1}^{\left(n_{1}\right)} \cap \cdots \cap P_{k}^{\left(n_{k}\right)}\right) B^{*} \subseteq A^{*}=R, \quad P_{1}^{\left(n_{1}\right)} \cap \cdots \cap P_{k}^{\left(n_{k}\right)} \subseteq B^{* *}=B
$$

and thus $B=P_{1}^{\left(n_{1}\right)} \cap \cdots \cap P_{k}^{\left(n_{k}\right)}$. The uniqueness of the decomposition will follow from the second part.

Since $B R_{P_{i}} \cap R$ is a reflexive ideal of $R$ by 2.1(1), it follows that $B R_{P_{i}} \cap R$ $=P_{i}^{\left(m_{i}\right)}$ for some $i$. Clearly $m_{i}=n_{i}$.

3. Primary decomposition. If $M$ is a right $R$-module, the associated primes of $M$, Ass $M$ consist of all prime ideals $P$ of $R$ satisfying the following property: there exists a submodule $N$ of $M$ such that $P$ is the annihilator of all nonzero submodules of $N$. Ass $M$ is the set of primes associated with $M$ in the sense of the Lesieur-Croisot tertiary decomposition theory. For $x \in R, N x=0$ for some submodule of $M$ if and only if $x$ belongs to some $P \in$ Ass $M$. Such an 
$x$ is called an annihilating element of $M$. Further properties of the LesieurCroisot theory and axiomatic decomposition theory can be found in Riley [65].

When $I$ is a right ideal of $R$, a finite set $\left\{I_{i}, i=1, \ldots, n\right\}$ of right ideals of $R$ each containing $I$ is called a tertiary decomposition for $I$ if $I=\bigcap_{j=1}^{n} I_{j}$ and Ass $\left(R / I_{i}\right)=\left\{P_{i}\right\}$ for each $i$. The decomposition is called reduced if $P_{i} \neq P_{j}, \forall i$ $\neq j$, and primary if, in addition, each $I_{j}$ contains some power of $P_{j}$.

Lemma 3.1. Let I be a reflexive essential right ideal of $R$. Then each associated prime of $R / I$ is reflexive.

Proof. Let $P \in$ Ass $(R / I)$. Then $I^{\prime} P \subseteq I$ for some right ideal $I^{\prime} \supsetneqq I$. If $P^{*}=R$, then $\left(I^{\prime} P\right)^{*}=I^{\prime *} \Rightarrow I^{\prime} \subseteq I^{\prime * *}=\left(I^{\prime} P\right)^{* *} \subseteq I^{* *}=I$, a contradiction. Thus, $P^{*} \supsetneqq R$ implying that $P$ is reflexive by Cozzens and Sandomierski [75].

THEOREM 3.2. Let I be a bounded, essential right ideal of $R$ with $B=$ bound $I$. Then:

(1) $I$ is reflexive if and only if the associated primes of $R / I$ are all reflexive.

(2) If $I$ is reflexive, $B$ is reflexive and Ass $R / I=$ Ass $R / B=\left\{P_{1}, \ldots, P_{m}\right\}$ where $B=\bigcap_{i=1}^{m} P_{i}^{\left(n_{i}\right)}$.

(3) When $I$ is reflexive, the set $\left\{I_{P_{i}} \cap R, i=1, \ldots, m\right\}$ is a reduced primary decomposition for $I$.

Proof. (1) Since Ass $\left(I^{* *} / I\right) \subseteq$ Ass $(R / I) \subseteq$ Ass $\left(I^{* *} / I\right) \cup$ Ass $\left(R / I^{* *}\right)$, the associated primes of $I^{* *} / I$ are reflexive if and only if the primes of $R / I$ are reflexive by 3.1. Since $I_{P}^{* *}=I_{P}, \forall P \in \mathcal{P}, I^{* *}=I$ if and only if the primes of $R / I$ are reflexive.

(2) That $B$ is reflexive when $I$ is reflexive, is trivial. Let $x$ be an annihilating element of $R / B$, then $B^{\prime} x \subseteq B$ for a two-sided ideal $B^{\prime} \supsetneq B$. Since $B^{\prime} \nsubseteq I, x$ is an annihilating element for $R / I \Rightarrow x$ belongs to some $P \in$ Ass $(R / I)$. Since the primes in Ass $(R / I)$ are minimal, Ass $(R / B) \subseteq$ Ass $(R / I)$. Conversely, if $P \in \operatorname{Ass}(R / I), I_{P} \neq R_{P} \Rightarrow B_{P} \neq R_{P} \Rightarrow P \in$ Ass $(R / B)$.

That Ass $(R / B)=\left\{P_{1}, \ldots, P_{m}\right\}$ when $B=\bigcap_{i=1}^{m} P_{i}^{\left(n_{i}\right)}$ is clear.

(3) If $I_{i}=I R_{P_{i}} \cap R, i=1, \ldots, m$, each $I_{i}$ is reflexive by $2.1(1)$, Ass $\left(R / I_{i}\right)$ $=\left\{P_{i}\right\}$ and $I=\cap I_{i}$ since $I$ is reflexive.

NOTE ADDED IN PROOF. It has been brought to the attention of the authors that there is some overlap between this paper and that of M. Chamarie, Localisations dans les ordres maximaux, Comm. Algebra (4) 2 (1974), 279-293.

\section{REFERENCES}

72. A. W. Chatters and S. M. Ginn, Localisation in hereditary rings, J. Algebra 22 (1972), 82-88. MR 46 \# 7282.

75. J. H. Cozzens and F. L. Sandomierski, Maximal orders and localization. I, J. Algebra (to appear).

67. A. W. Goldie, Localization in non-commutative noetherian rings, J. Algebra 5 (1967), 89-105. MR 34 \# 7562; erratum, 35, p. 1577. 
71. C. R. Hajarnavis and T. H. Lenagan, Localisation in Asano orders, J. Algebra 21 (1972), 441-449. MR 46 \#9090.

72. J. Kuzmanovich, Localizations of Dedekind prime rings, J. Algebra 21 (1972), 378-393. MR $47 \# 260$.

68. J. C. McConnell, Localisations in enveloping rings, J. London Math. Soc. 43 (1968), 421-428. MR 37 \#4112.

69. G. O. Michler, Asano orders, Proc. London Math. Soc. (3) 19 (1969), 421-443. MR 40 \#188.

72. - Right symbolic powers and classical localization in right Noetherian rings, Math. Z. 127 (1972), 57-69. MR 46 \#9106.

65. John A. Riley, Reflexive ideals in maximal orders, J. Algebra 2 (1965), 451-465. MR 32 \#7585.

68. J. C. Robson, Non-commutative Dedekind rings, J. Algebra 9 (1968), 249-265. MR 38 \#177.

Department of Mathematics, Rider College, Trenton, New Jersey 08602

Department of Mathematics, Kent State University, Kent, Ohio 44242 